WIS/05/06-APR-DPP

TAUP-2824/06

hep-th/0604161

\title{
A Holographic Model of Deconfinement and Chiral Symmetry Restoration
}

\author{
Ofer Aharony $^{a}$, Jacob Sonnenschein $^{b, c}$, and Shimon Yankielowicz ${ }^{b, c}$ \\ ${ }^{a}$ Department of Particle Physics \\ The Weizmann Institute of Science, Rehovot 76100, Israel \\ ${ }^{b}$ School of Physics and Astronomy \\ The Raymond and Beverly Sackler Faculty of Exact Sciences \\ Tel Aviv University, Ramat Aviv 69978, Israel \\ ${ }^{c}$ Albert Einstein Minerva Center \\ The Weizmann Institute of Science, Rehovot 76100, Israel
}

\begin{abstract}
We analyze the finite temperature behavior of the Sakai-Sugimoto model, which is a holographic dual of a theory which spontaneously breaks a $U\left(N_{f}\right)_{L} \times U\left(N_{f}\right)_{R}$ chiral flavor symmetry at zero temperature. The theory involved is a $4+1$ dimensional supersymmetric $S U\left(N_{c}\right)$ gauge theory compactified on a circle of radius $R$ with anti-periodic boundary conditions for fermions, coupled to $N_{f}$ left-handed quarks and $N_{f}$ right-handed quarks which are localized at different points on the compact circle (separated by a distance $L$ ). In the supergravity limit which we analyze (corresponding in particular to the large $N_{c}$ limit of the gauge theory), the theory undergoes a deconfinement phase transition at a temperature $T_{d}=1 / 2 \pi R$. For quark separations obeying $L>L_{c} \simeq 0.97 * R$ the chiral symmetry is restored at this temperature, but for $L<L_{c} \simeq 0.97 * R$ there is an intermediate phase which is deconfined with broken chiral symmetry, and the chiral symmetry is restored at $T_{\chi S B} \simeq 0.154 / L$. All of these phase transitions are of first order.
\end{abstract}

E-mails : Ofer.Aharony@weizmann.ac.il, cobi@post.tau.ac.il, shimonya@post.tau.ac.il. 


\section{Introduction}

QCD, the theory of the strong nuclear interactions, exhibits two interesting phenomena at low energies - confinement and (approximate) spontaneous chiral symmetry breaking. Both of these phenomena are strong coupling effects which are not visible in perturbation theory, and there are no known quantitative methods to study them (except by lattice simulations). Apriori there is no relation between these two phenomena, and there are known examples of chiral symmetry breaking in non-confining theories and of confinement without chiral symmetry breaking. However, in QCD both phenomena occur together.

New methods for studying strongly coupled gauge theories have been developed in the last decade, following the AdS/CFT correspondence [1. These methods are particularly powerful in large $N_{c}$ gauge theories which are dual to weakly coupled string theories in weakly curved spaces, since then many computations can be explicitly performed. This class of theories does not include (large $N_{c}$ ) QCD, but there are several known cases of theories which are labeled by a dimensionless parameter, such that in one limit of the parameter the string background is weakly curved, while the other limit gives (large $N_{c}$ ) QCD [2, 3, 4, 5]. It is believed that these theories are (for any value of the dimensionless parameter) in the same universality class as QCD, so it is interesting to try to use these theories to understand better the phenomena of confinement and chiral symmetry breaking.

Understanding confinement in the weakly curved limit is amazingly easy; the confining strings are explicitly seen as strings in the weakly curved background (either fundamental strings or strings arising from D-branes), and their tensions are easily computable. It would be nice to be able to similarly study the phenomenon of chiral symmetry breaking $(\chi \mathrm{SB})$, and to see if (in the weakly curved limit) there is any link between $\chi \mathrm{SB}$ and confinement. Unfortunately, it seems much more difficult to find dual models for theories with non-Abelian $U\left(N_{f}\right)_{L} \times U\left(N_{f}\right)_{R}$ chiral symmetry than to find dual models for confining theories. In the context of holographic dual models, quarks in the fundamental representation of $S U\left(N_{c}\right)$ were first incorporated in terms of probe $\mathrm{D}$ branes in [6] ${ }^{1}$. Since then several holographic models dual to theories with quarks have been constructed and investigated, with the quarks coming from additional "flavor D-branes", in both conformal and confining gauge theories [8, 9, 10, 11, 12, 13, 14, 15. However, there are relatively few brane constructions with non-Abelian chiral symmetry (see, e.g., [16]), and it has proven difficult to use these brane constructions to construct (by taking a near-horizon limit) string theory duals of theories with non-Abelian chiral symmetry (with or without $\chi \mathrm{SB})^{2}$.

\footnotetext{
${ }^{1}$ The first appearance of quarks in the fundamental representation of $\operatorname{Sp}\left(N_{c}\right)$ was in [7].

${ }^{2}$ On the other hand, there are several models realizing a $U(1)$ axial flavor symmetry, such as 9,10 .
} 
This situation was recently changed by the work of Sakai and Sugimoto [17, 18, who found a simple brane construction realizing chiral symmetry, and took its near-horizon limit to find a string theory dual to a field theory with chiral symmetry breaking. The brane construction is in type IIA string theory compactified on a circle of radius $R$ in the $x_{4}$ direction, and involves $N_{c}$ D4-branes wrapped on the circle (filling the 01234 directions), $N_{f}$ D8-branes sitting at the point $x_{4}=0$ and $N_{f}$ anti-D8-branes sitting at the point $x_{4}=L^{3}$. The circle could have either periodic or anti-periodic boundary conditions for the fermions; the more interesting case for relating to QCD is the case in which all fermions have anti-periodic boundary conditions around the circle. The gauge theory on the D4-branes at low energies is a $U\left(N_{c}\right) 4+1$ dimensional maximally supersymmetric Yang-Mills theory, which is coupled to $N_{f}$ left-handed $3+1$-dimensional chiral fermions (in the fundamental representation of $U\left(N_{c}\right)$ ) at the intersection with the D8-branes and to $N_{f}$ right-handed chiral fermions at the intersection with the anti-D8-branes. With anti-periodic boundary conditions, the fermions in the $4+1$ dimensional gauge multiplet obtain a mass of order $1 / R$, and the scalars obtain masses from quantum corrections, so the (classical) massless spectrum is that of a $3+1$ dimensional $U\left(N_{c}\right)$ gauge theory coupled to $N_{f}$ massless Dirac fermions. The $U\left(N_{f}\right)_{L} \times U\left(N_{f}\right)_{R}$ global chiral symmetry of this theory ${ }^{4}$ is explicitly visible as the gauge symmetry on the D8 and anti-D8-branes.

The string theory described above is non-supersymmetric and unstable, but this instability does not affect the low-energy behavior, and disappears when we take a decoupling limit for the theory on the D4-branes; the unstable modes, which are the scalar fields corresponding to the radius of the circle and to the asymptotic brane-anti-brane separation, become non-normalizable in this near-horizon limit so we do not need to worry about them. This decoupling limit, before the compactification on a circle, was described in [19, and it involves taking the string scale $\alpha^{\prime}$ to zero and the asymptotic string coupling $g_{s}$ to infinity while keeping fixed the $4+1$ dimensional gauge coupling $g_{5}^{2} \sim g_{s} \sqrt{\alpha^{\prime}}$. The fact that the (asymptotic) string coupling goes to infinity in this limit is related to the fact that the $4+1$ dimensional gauge theory is not well-defined at high energies, and should be defined with some UV completion; in type IIA string theory the completion defines this theory as the $5+1$-dimensional $\mathcal{N}=(2,0)$ superconformal theory of M5branes compactified on a circle, but this will not be relevant for our purposes (since we will always be interested in energy scales well below $1 / g_{5}^{2}$ ). The near-horizon limit of the D4-branes, which is dual to their (UV-completed) $4+1$-dimensional supersymmetric gauge theory, was described in [19]. It is highly curved at small radial coordinates (corresponding to the fact that the gauge theory is IR-free), and at very large radial

\footnotetext{
${ }^{3}$ In [17] the specific choice $L=\pi R$ was made, for which the D8's and anti-D8's are antipodal on the circle, but this is not really necessary.

${ }^{4}$ The axial $U(1)$ is anomalous, but this is a subleading effect in the large $N_{c}$ limit which will not be relevant for our leading order computations.
} 
coordinates the string coupling becomes large and the background needs to be lifted to M theory, but for large $N_{c}$ there is a large range of values of the radial coordinate for which type IIA string theory gives a good description.

The near-horizon limit of D4-branes compactified on a circle with anti-periodic boundary conditions was first discussed in [2], where it was noted that it was the same as the (Wick rotation of the) near-horizon limit of near-extremal D4-branes, and that when the radius of the circle is small compared to the scale of the 't Hooft coupling of the YangMills theory, the background becomes weakly curved everywhere. This was, in fact, the first model of a confining theory constructed following the AdS/CFT correspondence. In the limit of large $N_{c}$ with fixed $N_{f}$, the back-reaction of the D8-branes is small, and they can be treated as probes in this background. Sakai and Sugimoto noted that in this setup there is a very nice interpretation of chiral symmetry breaking ${ }^{5}$. At large radial positions (corresponding to the high-energy limit of the field theory) the D8-branes and anti-D8-branes are separated and one sees the full $U\left(N_{f}\right)_{L} \times U\left(N_{f}\right)_{R}$ chiral symmetry. Apriori, as we go into the interior of space, the D8-branes and the anti-D8-branes could remain separated, or they could join together into $N_{f}$ continuous 8-branes, so that only a single (diagonal) $U\left(N_{f}\right)$ symmetry is visible, corresponding to spontaneous chiral symmetry breaking. In the background of [2], the radial position has a minimal value (where the $x_{4}$-circle shrinks to zero size) and the 8-branes have nowhere to end, and therefore the D8-branes have to smoothly join the anti-D8-branes and exhibit $\chi \mathrm{SB}$. In [17] an explicit solution for the position of the 8-branes was constructed (in the probe approximation) which realizes this.

Recently, it was realized in [20] that the scale of chiral symmetry breaking in this model is actually independent of the scale of confinement (the first depends mostly on $L$ while the second depends mostly on $R$ ), and that chiral symmetry breaking persists even in the $R \rightarrow \infty$ limit in which confinement disappears ${ }^{6}$. We will see this separation of scales also in our finite temperature analysis.

In this paper we will study the model of Sakai and Sugimoto at finite temperature. In asymptotically free gauge theories with confinement and chiral symmetry breaking, both of these properties disappear at high temperatures as the effective coupling becomes small. In QCD there is no sharp phase transition distinguishing the low-temperature and high-temperature phases, since there are no sharp order parameters for confinement (due to the presence of dynamical quarks) and for the (approximate) chiral symmetry breaking. However, in a theory with exactly massless quarks one expects to have a sharp phase transition associated with the chiral symmetry restoration (characterized by the

\footnotetext{
${ }^{5} \mathrm{~A}$ similar structure of probe branes in a confining background was previously introduced in [8] in the context of the model of [4].

${ }^{6}$ The independence of the two phenomena in the gravity approximation was first noted for an Abelian chiral symmetry in [13].
} 
chiral condensate as an order parameter), and in the large $N_{c}$ limit the deconfinement transition also becomes a sharp phase transition (since the quarks can be ignored in this limit, and the pure gauge theory at finite temperature has a $Z_{N_{c}}$ global symmetry which is unbroken in the confined phase and broken in the deconfined phase). It is interesting to ask whether these two phase transitions occur at the same temperature or not. In the large $N_{c}$ limit of QCD this is a difficult question to answer analytically. However, in the Sakai-Sugimoto model (which is continuously related to large $N_{c}$ QCD by varying parameters) we will see that the question is easy to answer ${ }^{7}$. We will find that simple geometrical arguments imply that chiral symmetry is always broken in the confined phase, so that chiral symmetry restoration can happen either at the same temperature as deconfinement or above it. A more detailed analysis will be necessary to decide between these two possibilities, and we will find that in the weakly-curved limit the answer to this question depends on the ratio $L / R$ (which can go between zero and $\pi$; in the limit of small $L / R$ this ratio is inversely related to the ratio between the low-spin meson masses and the low-spin glueball masses). We will show that for $L / R>0.97$ the two phase transitions occur together, while for $L / R<0.97$ the chiral symmetry restoration transition happens at a higher temperature than the deconfinement transition. In the supergravity limit all of these phase transitions are of first order.

We begin in section 2 with a description of the system at zero temperature. In particular we present a short review of the Sakai-Sugimoto model and its meson spectrum, generalizing the construction of [17] to arbitrary values of $L / R$. In section 3 we discuss the finite temperature behavior of this theory. We start by discussing the deconfinement transition, which is associated with the bulk physics, and its implications on chiral symmetry breaking. We continue by studying the realization of chiral symmetry in the deconfined phase and computing the chiral symmetry restoration temperature. We also discuss the spectrum of quark-anti-quark excitations of the theory at finite temperature. We end in section 4 with our conclusions. An appendix contains the computation of the difference of free energies which is relevant for the deconfinement transition.

\section{The zero temperature string theory model}

The string theory dual of the gauge theory we described above at zero temperature is the Sakai-Sugimoto model [17]. We start by reviewing the basic structure of this model. We then describe the spectrum of mesons of low and high spins.

\footnotetext{
${ }^{7}$ Note that this theory is not asymptotically free, so apriori it is not clear whether at high temperatures it should deconfine and restore chiral symmetry or not.
} 


\subsection{Review of the Sakai-Sugimoto model}

The model of Sakai and Sugimoto [17] describes the near-horizon limit of a configuration of $N_{c}$ D4-branes, wrapping a circle in the $x_{4}$ direction with anti-periodic boundary conditions for the fermions, and intersected by $N_{f}$ D8-branes (located at $x_{4}=0$ ) and $N_{f}$ anti-D8-branes (located at $x_{4}=L$ ). This is dual to a $4+1$ dimensional maximally supersymmetric $S U\left(N_{c}\right)$ Yang-Mills theory (with coupling constant $g_{5}$ and with a specific UV completion that will not be important for us), compactified on a circle of radius $R$ with anti-periodic boundary conditions for the fermions, with $N_{f}$ left-handed quarks located at $x_{4}=0$ and $N_{f}$ right-handed quarks located at $x_{4}=L$ (obviously we can assume $L \leq \pi R)$.

In the limit $N_{f} \ll N_{c}$, this background can be described by $N_{f}$ probe D8-branes inserted into the near-horizon limit of a set of $N_{c}$ D4-branes compactified on a circle with anti-periodic boundary conditions for the fermions [2]. This background is simply related to the (near-horizon limit of the) background of near-extremal D4-branes by exchanging the roles (and signatures) of the time direction and of one of the spatial directions. Let us now briefly review this model, emphasizing the manifestations of confinement and chiral symmetry breaking. The background of type IIA string theory is characterized by the metric, the RR four-form and a dilaton given by

$$
\begin{aligned}
& d s^{2}=\left(\frac{u}{R_{D 4}}\right)^{3 / 2}\left[-d t^{2}+\delta_{i j} d x^{i} d x^{j}+f(u) d x_{4}^{2}\right]+\left(\frac{R_{D 4}}{u}\right)^{3 / 2}\left[\frac{d u^{2}}{f(u)}+u^{2} d \Omega_{4}^{2}\right], \\
& F_{(4)}=\frac{2 \pi N_{c}}{V_{4}} \epsilon_{4}, \quad e^{\phi}=g_{s}\left(\frac{u}{R_{D 4}}\right)^{3 / 4}, \quad R_{D 4}^{3} \equiv \pi g_{s} N_{c} l_{s}^{3}, \quad f(u) \equiv 1-\left(\frac{u_{\Lambda}}{u}\right)^{3},
\end{aligned}
$$

where $t$ is the time direction and $x^{i}(i=1,2,3)$ are the uncompactified world-volume coordinates of the D4 branes, $x_{4}$ is a compactified direction of the D4-brane worldvolume which is transverse to the probe D8 branes, the volume of the unit four-sphere $\Omega_{4}$ is denoted by $V_{4}$ and the corresponding volume form by $\epsilon_{4}, l_{s}$ is the string length and finally $g_{s}$ is a parameter related to the string coupling. The submanifold of the background spanned by $x_{4}$ and $u$ has the topology of a cigar (as on the left-hand side of figure 3 below) where the minimum value of $u$ at the tip of the cigar is $u_{\Lambda}$. The tip of the cigar is non-singular if and only if the periodicity of $x_{4}$ is

$$
\delta x_{4}=\frac{4 \pi}{3}\left(\frac{R_{D 4}^{3}}{u_{\Lambda}}\right)^{1 / 2}=2 \pi R
$$

and we identify this with the periodicity of the circle that the $4+1$-dimensional gauge theory lives on.

The parameters of this gauge theory, the five-dimensional gauge coupling $g_{5}$, the lowenergy four-dimensional gauge coupling $g_{4}$, the glueball mass scale $M_{g b}$, and the string 
tension $T_{s t}$ are determined from the background (11) in the following form [10]:

$$
\begin{aligned}
g_{5}^{2} & =(2 \pi)^{2} g_{s} l_{s}, \quad g_{4}^{2}=\frac{g_{5}^{2}}{2 \pi R}=3 \sqrt{\pi}\left(\frac{g_{s} u_{\Lambda}}{N_{c} l_{s}}\right)^{1 / 2}, \quad M_{g b}=\frac{1}{R} \\
T_{s t} & =\left.\frac{1}{2 \pi l_{s}^{2}} \sqrt{g_{t t} g_{x x}}\right|_{u=u_{\Lambda}}=\frac{1}{2 \pi l_{s}^{2}}\left(\frac{u_{\Lambda}}{R_{D 4}}\right)^{3 / 2}=\frac{2}{27 \pi} \frac{g_{4}^{2} N_{c}}{R^{2}}=\frac{\lambda_{5}}{27 \pi^{2} R^{3}},
\end{aligned}
$$

where $\lambda_{5} \equiv g_{5}^{2} N_{c}, M_{g b}$ is the typical scale of the glueball masses computed from the spectrum of excitations around (11), and $T_{s t}$ is the confining string tension in this model (given by the tension of a fundamental string stretched at $u=u_{\Lambda}$ where its energy is minimized). The gravity approximation is valid whenever $\lambda_{5} \gg R$, otherwise the curvature at $u \sim u_{\Lambda}$ becomes large. Note that as usual in gravity approximations of confining gauge theories, the string tension is much larger than the glueball mass scale in this limit. At very large values of $u$ the dilaton becomes large, but this happens at values which are of order $N_{c}^{4 / 3}$ (in the large $N_{c}$ limit with fixed $\lambda_{5}$ ), so this will play no role in the large $N_{c}$ limit that we will be interested in. The Wilson line of this gauge theory (before putting in the D8-branes) admits an area law behavior [21, as can be easily seen using the conditions for confinement of [22].

Naively, at energies lower than the Kaluza-Klein scale $1 / R$ the dual gauge theory is effectively four dimensional; however, it turns out that the theory confines and develops a mass gap of order $M_{g b}=1 / R$, so (in the regime where the gravity approximation is valid) there is no real separation between the confined four-dimensional fields and the higher Kaluza-Klein modes on the circle. As discussed in [2], in the opposite limit of $\lambda_{5} \ll R$, the theory approaches the $3+1$ dimensional pure Yang-Mills theory at energies small compared to $1 / R$, since in this limit the scale of the mass gap is exponentially small compared to $1 / R$. It is believed that there is no phase transition when varying $\lambda_{5} / R$ between the gravity regime and the pure Yang-Mills regime, but it is not clear how to check this.

Next, we introduce the probe 8 -branes which span the coordinates $t, x^{i}, \Omega_{4}$, and follow some curve $u\left(x_{4}\right)$ in the $\left(x_{4}, u\right)$-plane. Near the boundary at $u \rightarrow \infty$ we want to have $N_{f}$ D8-branes localized at $x_{4}=0$ and $N_{f}$ anti-D8-branes (or D8-branes with an opposite orientation) localized at $x_{4}=L$. Naively one might think that the D8-branes and antiD8-branes would go into the interior of the space and stay disconnected; however, these 8-branes do not have anywhere to end in the background (11), so the form of $u\left(x_{4}\right)$ must be such that the D8-branes smoothly connect to the anti-D8-branes (namely, $u$ must go to infinity at $x_{4}=0$ and at $x_{4}=L$, and $d u / d x_{4}$ must vanish at some minimal $u$ coordinate $u=u_{0}$ ). Such a configuration spontaneously breaks the chiral symmetry from the symmetry group which is visible at large $u, U\left(N_{f}\right)_{L} \times U\left(N_{f}\right)_{R}$, to the diagonal $U\left(N_{f}\right)$ symmetry. Thus, in this configuration the topology forces a breaking of the chiral 
symmetry; this is not too surprising since chiral symmetry breaking at large $N_{c}$ follows from rather simple considerations 23 .

In order to find the 8-brane configuration, we need the induced metric on the D8branes, which is

$$
\begin{aligned}
d s_{D 8}^{2} & =\left(\frac{u}{R_{D 4}}\right)^{3 / 2}\left[-d t^{2}+\delta_{i j} d x^{i} d x^{j}\right]+\left(\frac{u}{R_{D 4}}\right)^{3 / 2}\left[f(u)+\left(\frac{R_{D 4}}{u}\right)^{3} \frac{u^{\prime 2}}{f(u)}\right] d x_{4}^{2} \\
& +\left(\frac{R_{D 4}}{u}\right)^{3 / 2} u^{2} d \Omega_{4}^{2}
\end{aligned}
$$

where $u^{\prime}=d u / d x_{4}$. It is easy to check that the CS term in the D8-brane action does not affect the solution of the equations of motion. More precisely, the equation of motion of the gauge field has a classical solution of a vanishing gauge field, since the CS term includes terms of the form $C_{5} \wedge F \wedge F$ and $C_{3} \wedge F \wedge F \wedge F$. So, we are left only with the DBI action. Substituting the determinant of the induced metric and the dilaton into the DBI action, we obtain (ignoring the factor of $N_{f}$ which multiplies all the D8-brane actions that we will write) :

$$
S_{D B I}=T_{8} \int d t d^{3} x d x_{4} d^{4} \Omega e^{-\phi} \sqrt{-\operatorname{det}(\hat{g})}=\frac{\hat{T}_{8}}{g_{s}} \int d x_{4} u^{4} \sqrt{f(u)+\left(\frac{R_{D 4}}{u}\right)^{3} \frac{u^{\prime 2}}{f(u)}},
$$

where $\hat{g}$ is the induced metric (4) and $\hat{T}_{8}$ includes the outcome of the integration over all the coordinates apart from $d x_{4}$.

The simplest way to solve the equation of motion is by using the Hamiltonian of the action (5), which is conserved (independent of $x_{4}$ ) :

$$
\frac{u^{4} f(u)}{\sqrt{f(u)+\left(\frac{R_{D 4}}{u}\right)^{3} \frac{u^{\prime 2}}{f(u)}}}=\text { constant }=u_{0}^{4} \sqrt{f\left(u_{0}\right)}
$$

where on the right-hand side of the equation we assumed that there is a point $u_{0}$ where the profile $u\left(x_{4}\right)$ of the brane has a minimum, $u^{\prime}\left(u=u_{0}\right)=0^{8}$. We need to find the solution in which as $u$ goes to infinity, $x_{4}$ goes to the values $x_{4}=0, L$; this implies

$$
\int d x_{4}=2 \int \frac{d u}{u^{\prime}}=L
$$

with $u^{\prime}$ given (as a function of $u$ ) by (지) (note that $u$ is a double-valued function of $x_{4}$ in these configurations, leading to the factor of two in (17) ). The form of this profile of the D8 brane is drawn in figure 1(a). Plugging in the value of $u^{\prime}$ from (6) we find

$$
L=\int d x_{4}=2 \int_{u_{0}}^{\infty} \frac{d u}{u^{\prime}}=2 R_{D 4}^{3 / 2} \int_{u_{0}}^{\infty} d u \frac{1}{f(u) u^{3 / 2} \sqrt{\frac{f(u) u^{8}}{f\left(u_{0}\right) u_{0}^{8}}-1}}
$$

${ }^{8}$ This type of analysis was done previously for Wilson line configurations. See, for instance, [21]. 


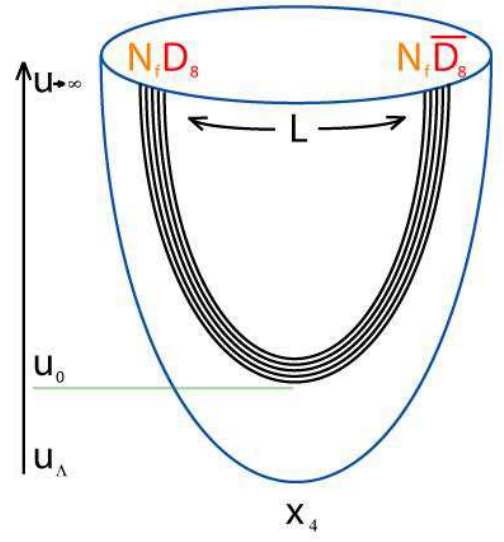

(a)

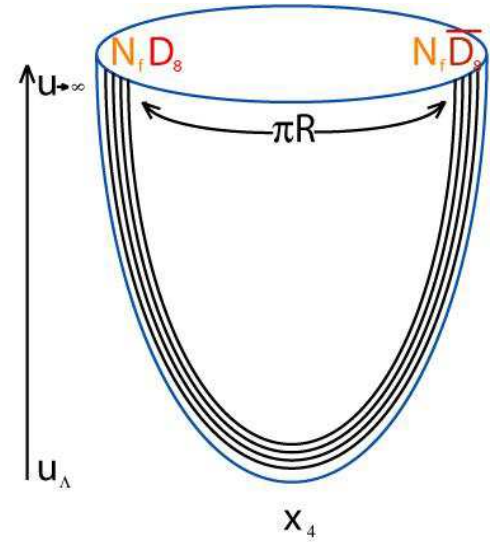

(b)

Figure 1: The dominant configurations of the D8 and anti-D8 probe branes in the SakaiSugimoto model at zero temperature, which break the chiral symmetry. The same configurations will turn out to be relevant also at low temperatures. On the left a generic configuration with an asymptotic separation of $L$, that stretches down to a minimum at $u=u_{0}$, is drawn. The figure on the right describes the limiting antipodal case $L=\pi R$, where the branes connect at $u_{0}=u_{\Lambda}$.

$$
=\frac{2}{3}\left(\frac{R_{D 4}^{3}}{u_{0}}\right)^{1 / 2} \sqrt{1-y_{\Lambda}^{3}} \int_{0}^{1} d z \frac{z^{1 / 2}}{\left(1-y_{\Lambda}^{3} z\right) \sqrt{1-y_{\Lambda}^{3} z-\left(1-y_{\Lambda}^{3}\right) z^{8 / 3}}}
$$

where $y_{\Lambda} \equiv u_{\Lambda} / u_{0}$. Small values of $L$ correspond to large values of $u_{0}$. In this limit we have $y_{\Lambda} \ll 1$ leading to $L \propto \sqrt{R_{D 4}^{3} / u_{0}}$. For general values of $L$ the dependence of $u_{0}$ on $L$ is more complicated.

There is a simple special case of the above solutions, which occurs when $L=\pi R$, namely the D8-branes and anti-D8-branes lie at antipodal points of the circle. In this case the solution for the branes is simply $x_{4}(u)=0$ and $x_{4}(u)=L=\pi R$, with the two branches meeting smoothly at the minimal value $u=u_{0}=u_{\Lambda}$ to join the D8-branes and the anti-D8-branes together. This type of antipodal solution is drawn in figure 1(b). It was shown in [17] that this classical configuration is stable, by analyzing small fluctuations around this configuration and checking that the energy density associated with them is non-negative.

For general $L$, one might apriori think that there could be two ways for the D8branes to connect to the anti-D8-branes - one in which they connect by curving towards 
positive $x_{4}$ (in the direction where the D8-anti-D8 distance is $L$ ) and the other by curving towards negative $x_{4}$ (in the direction where the distance is $2 \pi R-L$ ). However, smooth configurations only occur when the integral (17) is less than or equal to $\pi R$, so there is only one smooth configuration for every value of $L$.

Note that the Sakai-Sugimoto model has 3 dimensionful parameters : $\lambda_{5}, L$ and $R$, and gravity is reliable whenever $\lambda_{5} \gg R$. The physics depends on the two dimensionless ratios of these two parameters; we will see that in the gravity limit the mass of the (lowspin) mesons is related to $1 / L$ while the mass of the (low-spin) glueballs is related to $1 / R$. As discussed above, in the limit $\lambda_{5} \ll R$ this theory approaches (large $N_{c}$ ) QCD at low energies. This remains true also after adding the flavors, at least when $L$ is of order $R$ (note that the parameter $L$ is not visible in the four-dimensional low-energy effective action). However, this QCD limit is opposite from the supergravity limit, and there are no known reliable methods to analyze it.

\subsection{The spectrum of mesons}

The mesons of the Sakai-Sugimoto model are described by strings ending on the D8branes. It is convenient to separate the discussion to low-spin mesons and high-spin mesons. The former are described via modes of the massless fields living on the D8branes, whereas the latter are associated with string configurations that "fall" from the D8-branes down to the wall at $u=u_{\Lambda}$, stretch along the wall and then go back up again 12 .

Let us start with the low-spin mesons. These mesons correspond on the string theory side to the fluctuations of the massless fields on the probe branes. The fluctuations of the gauge fields on the branes give pseudo-vector and scalar mesons as well as massless "pions", and the fluctuations of the scalar field describing the embedding of the branes give massive scalar mesons. In [17] these two sectors of the mesonic spectrum were computed for the special case of $u_{0}=u_{\Lambda}$, namely $L=\pi R$. It is not difficult to generalize this analysis to general values of $L$ which we are interested in here, using the analysis of the fluctuations performed in [14] which we summarize below (the discussion in [14] is for a non-critical $A d S_{6}$ model, but one can easily apply the same analysis for our model).

As an example we describe in detail the modes coming from the components of the gauge field living on the D8-branes which are not in the $S^{4}$ directions. If we write the action using the coordinate $u$ (instead of using $x_{4}$ as in (5) ), the equations of motion for 
these fields that follow from the DBI action are given by ${ }^{9}$

$$
\begin{aligned}
u^{5 / 2} \gamma(u)^{1 / 2} \partial_{\mu} F^{\mu \nu}-\partial_{u}\left(u^{5 / 2} \gamma(u)^{1 / 2} F^{\nu u}\right) & =0, \\
\partial_{\mu} F^{\mu u} & =0
\end{aligned}
$$

where $\mu, \nu=0,1,2,3$ and where we have defined

$$
\gamma(u) \equiv \frac{u^{8}}{f(u) u^{8}-f\left(u_{0}\right) u_{0}^{8}} .
$$

We now separate variables and expand the five dimensional gauge fields in modes in the radial direction as follows :

$$
A_{\mu}\left(x^{\nu}, u\right)=\sum_{n} B_{\mu}^{(n)}\left(x^{\nu}\right) \psi_{n}(u), \quad A_{u}\left(x^{\nu}, u\right)=\sum_{n} \pi^{(n)}\left(x^{\nu}\right) \phi_{n}(u) .
$$

Using this decomposition, equation (10) reads:

$$
\sum_{n}\left(\tilde{m}_{n}^{2} \pi^{(n)}\left(x^{\nu}\right) \phi_{n}(u)-\left(\partial_{\mu} B_{(n)}^{\mu}\left(x^{\nu}\right)\right) \partial_{u} \psi_{n}(u)\right)=0
$$

where $\tilde{m}_{n}$ is the four-dimensional mass of the field $\pi^{(n)}$, namely $\eta^{\mu \nu} \partial_{\mu} \partial_{\nu} \pi^{(n)}=\tilde{m}_{n}^{2} \pi^{(n)}$. We can now choose a Lorentz gauge $\partial_{\mu} A^{\mu}=0 \rightarrow \partial_{\mu} B_{(n)}^{\mu}=0$, and in this gauge it is easy to see that all the $\pi^{(n)}$ fields are set to zero by (13) except for the Goldstone bosons $\pi^{(0)}$ with $\tilde{m}_{0}=0$, which survive as a massless field for any value of $u_{0}$. The other equation of motion (9) fixes the form of $\phi_{0}$ to be

$$
\phi_{0} \propto \frac{\gamma^{1 / 2}}{u^{5 / 2}}
$$

Finally, the eigenvalue equation for the modes $\psi_{n}(u)$ reads

$$
-u^{1 / 2} \gamma^{-1 / 2} \partial_{u}\left(u^{5 / 2} \gamma^{-1 / 2} \partial_{u} \psi_{n}\right)=R_{D 4}^{3} m_{n}^{2} \psi_{n}
$$

where $m_{n}$ are the masses of the four-dimensional vector fields $B_{\mu}^{(n)}$, such that their equation of motion is $\eta^{\nu \rho} \partial_{\nu} \partial_{\rho} B_{\mu}^{(n)}=m_{n}^{2} B_{\mu}^{(n)}$. Imposing appropriate orthonormality conditions [14, one finds that the effective four-dimensional action for these fluctuations of the gauge field living on the probe D8-branes can be written as:

$$
S=-\int d^{4} x \operatorname{tr}\left[\frac{1}{2} \partial_{\mu} \pi^{(0)} \partial^{\mu} \pi^{(0)}+\sum_{n \geq 1}\left(\frac{1}{4} F_{\mu \nu}^{(n)} F^{\mu \nu(n)}+\frac{1}{2} m_{n}^{2} B_{\mu}^{(n)} B^{\mu(n)}\right)\right] .
$$

${ }^{9}$ We perform the analysis for a single D8-brane, but the generalization to $N_{f}$ D8-branes is simply performed by turning all the fields into $N_{f} \times N_{f}$ matrices, turning the derivatives into $U\left(N_{f}\right)$-covariant derivatives, and taking a trace in the action. 
The fields $\pi^{(0)}$ are the Goldstone bosons associated with the spontaneous breaking of the $U\left(N_{f}\right)_{L} \times U\left(N_{f}\right)_{R}$ global symmetry to the diagonal $U\left(N_{f}\right)$ (which is the global symmetry of the remaining action (16)). As in [17, one can also go to the $A_{u}=0$ gauge, where there are no $\pi^{(n)}$ fields and the Goldstone bosons are encoded in the boundary value (as $u \rightarrow \infty$ ) of $A_{\mu}$. Note that the pions (the $\pi^{(0)}$ fields) are massless for any value of $u_{0}$ (or for any value of $L$ ), as expected since the quarks are massless (in the sense of having a vanishing current algebra mass) for any value of $L$.

The explicit mass spectrum of the vector mesons is found by looking for normalizable eigenfunctions of (15). In the special case of $u_{0}=u_{\Lambda}$ this spectrum was derived in [17. For generic probe profiles, for which $u_{0} \neq u_{\Lambda}$, the spectrum was computed in 14 for a similar non-critical string model. In general this spectrum is quite complicated. In the limit of $u_{0} \gg u_{\Lambda}$ or $L \ll R$ the spectrum simplifies. In this limit we can approximate $f(u) \simeq 1$, and there is a scaling symmetry of (15) which implies that all $m_{n}^{2}$ are proportional to $u_{0} / R_{D 4}^{3}$. Equation (8) then implies that this is proportional to $1 / L^{2}$, so in this limit all the low-spin meson masses are simply proportional to $1 / L$. This illustrates the separation of scales between the low-spin mesons and the low-spin glueballs (whose masses are proportional to $1 / R$ in the supergravity approximation) in the Sakai-Sugimoto model (as emphasized in [20]).

The DBI action of the D8-branes only includes mesons of spin one or less. To describe higher-spin mesons we need to look at more general string configurations that start and end on the probe branes. For large spin these strings are long and can be described semiclassically. The relevant string configurations can be schematically decomposed into three parts : a segment from the probe brane (at $\left.u=u_{0}\right)$ to the wall $\left(u=u_{\Lambda}\right)$, then a segment that stretches along the wall in the spatial directions, and then another "vertical" part stretching from the wall back to the probe brane, as depicted in figure 2 .

By computing the energy of the string it was shown in 12 that this string is equivalent to a string in flat space-time with two massive endpoints. The mass of the end-points, which can be identified with the constituent quark mass $m_{q}^{C}$, is given by [14]

$$
m_{q}^{C}=\frac{1}{2 \pi \alpha^{\prime}} \int_{u_{\Lambda}}^{u_{0}} \sqrt{-g_{t t} g_{u u}} d u=\frac{1}{2 \pi \alpha^{\prime}} \int_{u_{\Lambda}}^{u_{0}} f^{-\frac{1}{2}}(u) d u,
$$

which is simply the mass of a string stretched from the D8-branes to the minimal value of $u$. Note that for small $L$ this constituent mass scales as $u_{0} / \alpha^{\prime} \propto \lambda_{5} / L^{2}$. Let us emphasize again that the bare mass and current algebra masses are always zero in our configurations, and there is no simple description of the deformation associated with turning on a bare mass for the quarks (which corresponds to turning on a non-normalizable mode of the string connecting the D8-branes to the anti-D8-branes). Using the map to the string with massive endpoints it is straightforward to calculate the classical mass and angular momentum of these stringy mesons [12, 24. Somewhat surprisingly, it was found in [14] 


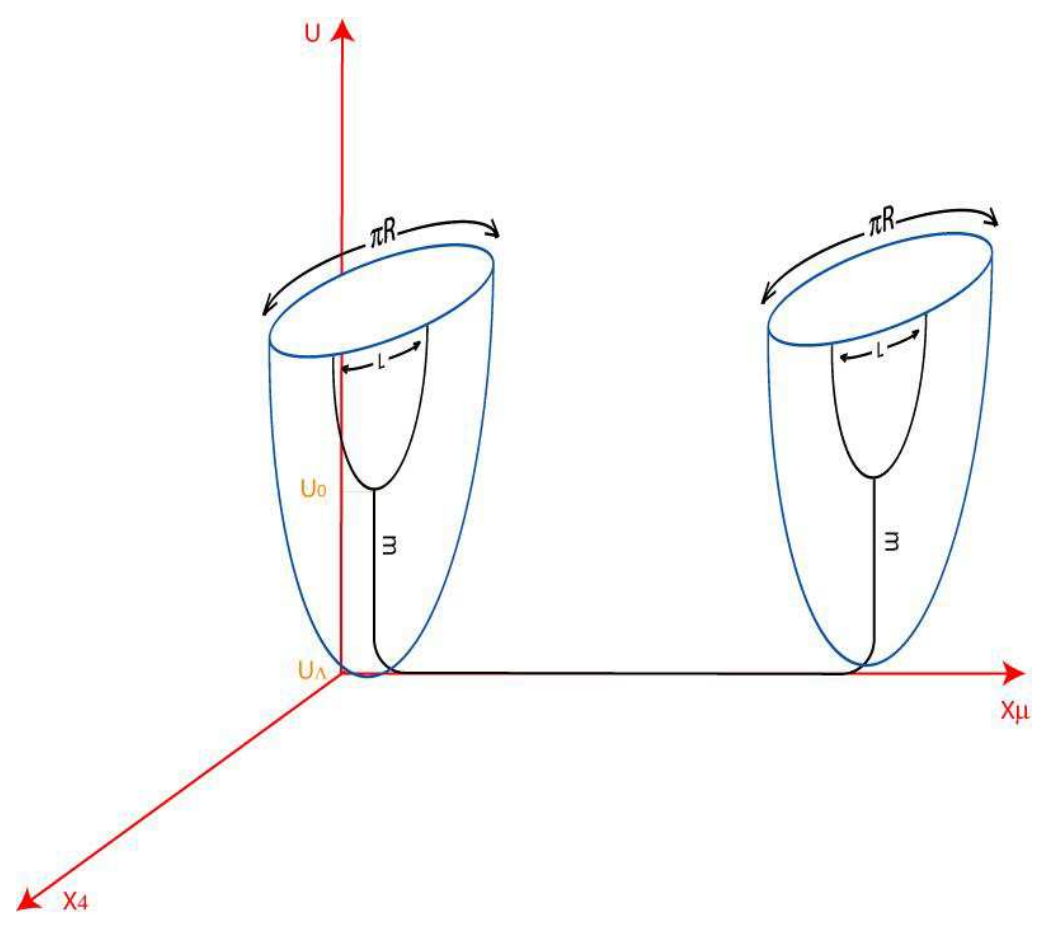

Figure 2: The high-spin "stringy" meson is a string starting at the lowest point of the probe branes $u=u_{0}$, going down to the wall, stretching horizontally in space along the wall, and then going back up vertically to the probe branes at $u=u_{0}$.

(in a similar model to the one we describe) that for $u_{0}$ of order $u_{\Lambda}$ there is a range of values of $u_{0} / u_{\Lambda}$ for which the masses of the light vector and pseudo scalar mesons scale roughly linearly with $m_{q}^{C}$, reinforcing its identification as a constituent quark mass. The decay processes of the stringy mesons were discussed in [15].

\section{Thermodynamics of the Sakai-Sugimoto model}

\subsection{Review of the bulk thermodynamics}

In the large $N_{c}$ limit (with finite $N_{f}$ ), the effect of the D8-branes on the background is subleading; the difference in free energies between different bulk backgrounds is of order $N_{c}^{2}$, while the contribution of the D8-branes is of order $N_{c} * N_{f}$. Thus, we can analyze the bulk thermodynamics independently of the presence of the D8-branes, and then add them in as probes to the dominant bulk background at each temperature.

The thermodynamical behavior of the bulk is analogous to the thermodynamical be- 

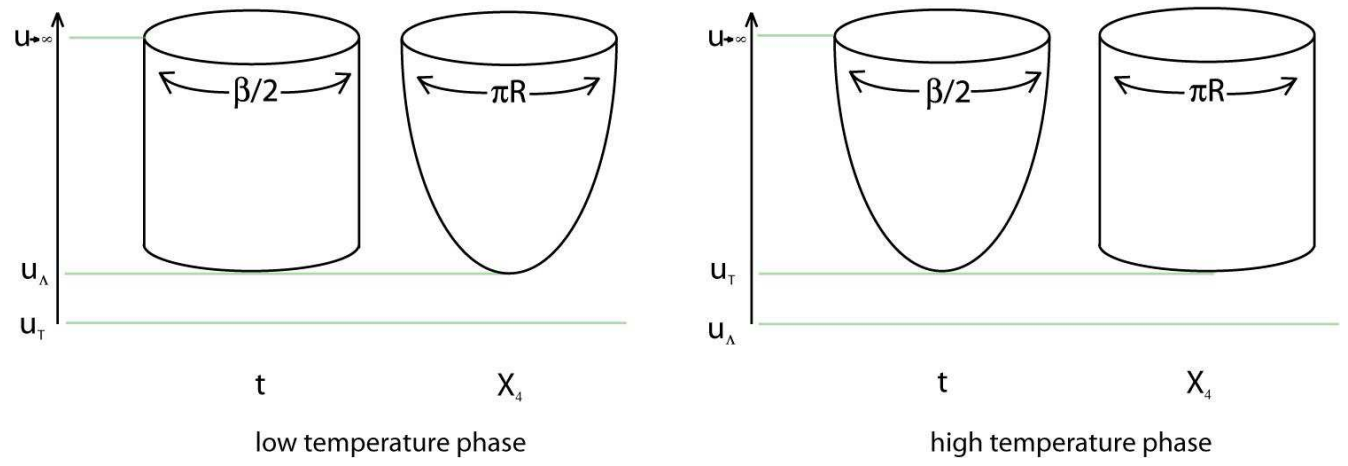

Figure 3: The topology of the solutions which dominate in the low temperature (confined) and high temperature (deconfined) phases of the Sakai-Sugimoto model, as reflected in the $(t, u)$ and $\left(x_{4}, u\right)$ submanifolds.

havior of other theories compactified on circles with anti-periodic boundary conditions for fermions in the gravity approximation (see, e.g., [2, 25]). In the gravity approximation, in order to study the theory at a finite temperature $T$, we need to look at Euclidean gravitational backgrounds which are asymptotically equal to (11), but with the time direction Euclidean and identified with a periodicity $\beta=1 / T$, and with anti-periodic boundary conditions for the fermions along this direction (as well as along the $x_{4}$ direction). One such background is obviously given by the Euclidean continuation of (1) itself, with $t$ periodically identified; it is easy to verify that this is a smooth background which is weakly curved (at least until the temperature reaches a number of order the square root of the confining string tension $T_{s t} \simeq \lambda_{5} / R^{3}$, at which stage the size of the time circle at the minimal radial position $u=u_{\Lambda}$ becomes of order the fundamental string scale and a tachyon develops). In this background the periodicity of $t$ is arbitrary and equal to $\beta$, and the periodicity of $x_{4}$ is $2 \pi R$ with $R$ related to $u_{\Lambda}$ by (2).

In the background described above the $x_{4}$ circle shrinks when $u$ reaches its minimal value, but the $t$ circle never shrinks to zero size. Another background with the same asymptotics is given by exchanging the behavior of the $t$ and $x_{4}$ circles, or equivalently by moving the factor of $f(u)$ in (11) from the $d x_{4}^{2}$ term to the $d t^{2}$ term. Now, the $t$ circle shrinks to zero size at the minimal value of $u$ (which is now related to $\beta$ rather than to $2 \pi R$, and given by $u_{T}=16 \pi^{2} R_{D 4}^{3} / 9 \beta^{2}$ ), while the $x_{4}$ circle never shrinks. This background also exists as a classical solution for any value of the periodicities of $x_{4}$ and $t$ (and develops a tachyon for very small values of $R$ ).

These two backgrounds are the only known smooth Euclidean solutions which asymp- 
totically approach (11) ${ }^{10}$. Thus, in order to decide which background dominates at a given temperature $T$ we need to compute the free energies of these backgrounds, given (in the gravitational approximation) by the classical supergravity action times the temperature, and see which one has a lower free energy. As usual, the classical action actually diverges and needs to be regulated. One way to regulate it is by looking at the difference between the actions of the two solutions, which turns out to be finite (this method is not always reliable, but it gives the correct answer in this case). Another way is to add counter-terms at a large $u$ cutoff that make the action finite as the cutoff goes to infinity. In the first method it is clear that the free energies of the two solutions agree when the (asymptotic) circumferences of the two circles are equal, $\beta=2 \pi R$, since the two solutions are identical then except for a relabeling of the coordinates. Thus, at this temperature $T_{d}=1 / 2 \pi R$ there is a first order phase transition between the two backgrounds (the transition is of first order since the solutions do not smoothly connect there, but continue to exist as separate solutions both below and above the transition). It is easy to see that the background in which the $x_{4}$ circle shrinks to zero size dominates at low temperatures $T<1 / 2 \pi R$, while the background in which the $t$ circle shrinks dominates at high temperatures, $T>1 / 2 \pi R$; intuitively, the circle which is smaller prefers to shrink to zero size. The relevant computation is described in the appendix, and leads (39) to a difference of free energy densities proportional to $N_{c}^{2} R\left(g_{s} N_{c}\right)\left[(2 \pi T)^{6}-1 / R^{6}\right]$.

The physical interpretation of this phase transition (which is a close relative of the Hawking-Page transition in asymptotically AdS backgrounds [2]) is straightforward. If we compute the quark-anti-quark potential in the two backgrounds (before adding the D8-branes) using the methods of [26, 27, 22], we find that in the low-temperature background in which $\sqrt{g_{t t} g_{x x}}$ is finite at the minimal value of $u$ the potential is linear in the distance, corresponding to confinement, while in the high-temperature background in which $\sqrt{g_{t t} g_{x x}}$ goes to zero at the minimal value of $u$ the potential decays with the distance, corresponding to a deconfined phase. Similarly, a computation of the value of the free energy in the two phases (which requires adding appropriate counter-terms) yields a result of order $N_{c}^{0}$ in the low-temperature phase, and a result of order $N_{c}^{2}$ in the high-temperature phase.

As mentioned above, as we increase $R$ compared to the scale set by the five dimensional gauge coupling $\lambda_{5}$, the supergravity background becomes highly curved, and the theory at low energies approaches the four dimensional pure Yang-Mills theory. It is believed (though it is difficult to prove) that the deconfinement transition described above

\footnotetext{
${ }^{10}$ Note that asymptotically these backgrounds become strongly coupled so we should really lift them to $\mathrm{M}$ theory, and discuss the asymptotics in the $\mathrm{M}$ theory regime, but in the large $N_{c}$ limit the strong coupling region can be arbitrarily far away from the regions of finite $u$ which we are interested in so this does not play any role.
} 


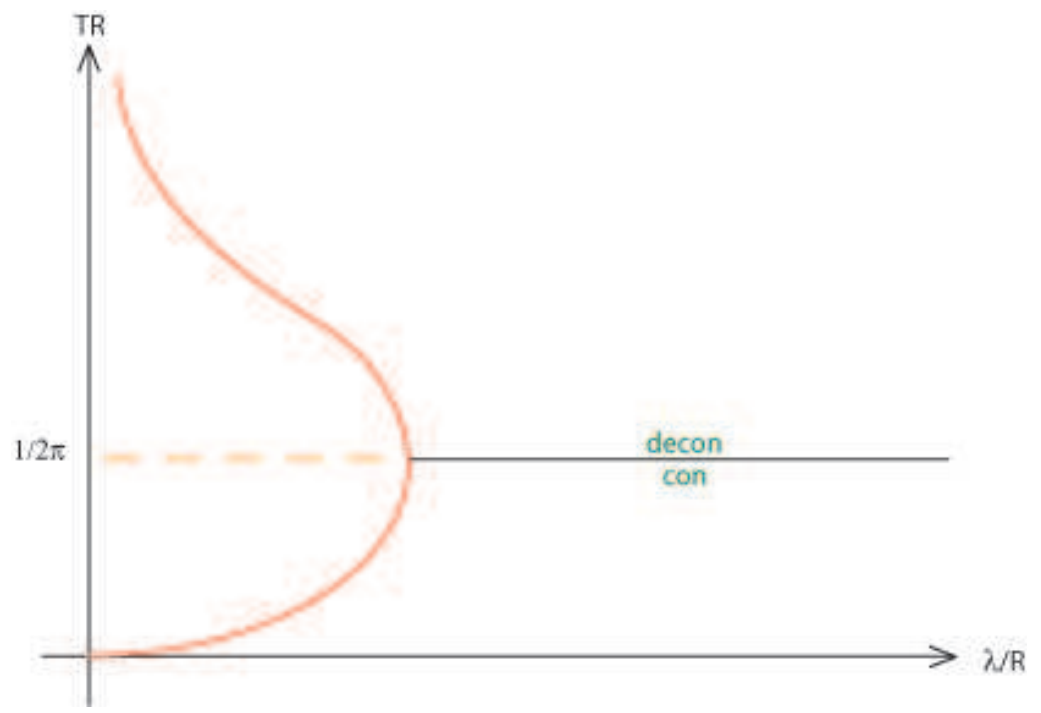

Figure 4: A minimal conjecture for the schematic form of the phase diagram of the $4+1$ dimensional maximally supersymmetric $S U\left(N_{c}\right)$ gauge theory compactified on a circle with anti-periodic boundary conditions for the fermions, as a function of the dimensionless coupling constant $\lambda_{5} / R$ and the dimensionless temperature $T R$, based on the known results in the various limits and on the $T \leftrightarrow 1 / 2 \pi R$ symmetry. All solid lines in the diagram denote phase transitions; the dashed line may or may not be a phase transition line.

is connected to the deconfinement transition of the large $N_{c}$ pure Yang-Mills theory in this limit. However, this connection is somewhat subtle [28, since even for small $\lambda_{5} / R$ it is clear by the symmetry arguments described above that there is always either a phase transition line at $T=1 / 2 \pi R$, or else the dominant phase at this temperature must be invariant under the exchange $T \leftrightarrow 1 / 2 \pi R$ (which is not the case for the confined and deconfined phases discussed above). On the other hand, the deconfinement phase transition in the pure Yang-Mills theory occurs at a temperature of order $\Lambda_{Q C D} \ll 1 / R$. Due to the $T \leftrightarrow 1 / 2 \pi R$ symmetry there must then be at least one more phase transition line at a temperature much larger than $1 / R$. Thus, we see that new phases must appear as we approach the four dimensional Yang-Mills limit. The phase structure of this theory in that limit includes several phase transition lines, which, presumably, all join into the line $T=1 / 2 \pi R$ in the gravity limit. A minimal conjecture for the phase structure is depicted in figure 4 ; note that with this conjecture the deconfinement transition line in the supergravity limit is connected to the deconfinement transition line in the four 
dimensional Yang-Mills limit, but this connection does not seem to be smooth ${ }^{11}$.

\subsection{The low temperature phase}

As described above, the background corresponding to the low-temperature phase of the theory is identical to that of the Sakai-Sugimoto model at zero temperature (11), apart from the fact that the time direction is Euclidean and compactified with a circumference $\beta=1 / T$, where $T$ is the temperature of the dual gauge theory. Hence, just as for the zero temperature case, the dual gauge theory is in the confining phase and the string tension is given by (3). Furthermore, the solution for the profile of the D8 brane is still given by (6). Note that this agrees with general arguments that the chiral condensate (in the large $N_{c}$ limit) should be independent of the temperature in the confined phase [30, 31. Defining $y \equiv u / u_{0}, y_{\Lambda} \equiv u_{\Lambda} / u_{0}$, and $f(y) \equiv 1-\left(y_{\Lambda} / y\right)^{3}$ (such that $f(1)=1-y_{\Lambda}^{3}$ ), we find from (6) that

$$
u^{\prime}=\left(\frac{u}{R_{D 4}}\right)^{3 / 2} f(y) \sqrt{y^{8} \frac{f(y)}{f(1)}-1} .
$$

Substituting this outcome of the equation of motion, the action is now

$$
\begin{aligned}
S_{D B I} & =2 \frac{\hat{T}_{8} R_{D 4}^{3 / 2} u_{0}^{7 / 2}}{g_{s}} \int_{1}^{\infty} d y \frac{y^{5 / 2}}{\sqrt{f(y)}} \frac{1}{\sqrt{1-\frac{f(1)}{f(y)} \frac{1}{y^{8}}}} \\
& =\frac{2 \hat{T}_{8} R_{D 4}^{3 / 2} u_{0}^{7 / 2}}{3 g_{s}} \int_{0}^{1} d z \frac{1}{z^{13 / 6} \sqrt{1-\left(1-y_{\Lambda}^{3}\right) z^{8 / 3}-y_{\Lambda}^{3} z}} .
\end{aligned}
$$

The form of the profile (18) of the D8-branes is drawn in figure प(a). Again, the topology of the background forces a profile that smoothly connects the D8-branes to the anti-D8 branes, signaling chiral symmetry breaking in the dual gauge theory.

The relation between $L$ and $u_{0}$ is precisely the same as for zero temperature (8). For small values of $L$ we find that the dependence of the action (19), which is proportional to the vacuum energy of the system, on the separation distance is given by

$$
S_{D B I} \propto \frac{\hat{T}_{8} R_{D 4}^{3 / 2}}{g_{s} L^{7}}
$$

For general values of $L$ the dependence is more complicated.

\footnotetext{
${ }^{11}$ This conjectured phase diagram is the same as the phase diagram of the same theory reduced on the three spatial directions which was discussed in [29], suggesting that perhaps the phase structure does not change when the three infinite spatial dimensions are compactified on a torus. We thank M. Van Raamsdonk for discussions of this point.
} 


\subsection{The high temperature phase}

The gravity background associated with the high temperature phase of the dual gauge theory takes the form

$$
\begin{aligned}
& d s^{2}=\left(\frac{u}{R_{D 4}}\right)^{3 / 2}\left[f(u) d t^{2}+\delta_{i j} d x^{i} d x^{j}+d x_{4}^{2}\right]+\left(\frac{R_{D 4}}{u}\right)^{3 / 2}\left[u^{2} d \Omega_{4}^{2}+\frac{d u^{2}}{f(u)}\right], \\
& F_{(4)}=\frac{2 \pi N_{c}}{V_{4}} \epsilon_{4}, \quad e^{\phi}=g_{s}\left(\frac{u}{R_{D 4}}\right)^{3 / 4}, \quad R_{D 4}^{3} \equiv \pi g_{s} N_{c} l_{s}^{3}, \quad f(u) \equiv 1-\left(\frac{u_{T}}{u}\right)^{3},
\end{aligned}
$$

in the same notations as in (11). The time direction $t$ now shrinks to zero size at the minimal value of $u, u=u_{T}$, and in order to avoid a singularity there the time direction must have a period of

$$
\delta t=\frac{4 \pi}{3}\left(\frac{R_{D 4}^{3}}{u_{T}}\right)^{1 / 2}=\beta
$$

which we identify with the inverse temperature of the system $\beta=1 / T$. On the other hand, in this solution the $x_{4}$ direction can have an arbitrary periodicity $2 \pi R$.

We expect that again the $\mathrm{D} 8$ probe branes span the coordinates $t, x^{i}, \Omega_{4}$, and are described by some curve $u\left(x_{4}\right)$. The induced metric on the $\mathrm{D} 8$ branes now reads

$$
\begin{aligned}
d s_{D 8}^{2} & =\left(\frac{u}{R_{D 4}}\right)^{3 / 2}\left[f(u) d t^{2}+\delta_{i j} d x^{i} d x^{j}\right]+\left(\frac{u}{R_{D 4}}\right)^{3 / 2}\left[1+\left(\frac{R_{D 4}}{u}\right)^{3} \frac{u^{\prime 2}}{f(u)}\right] d x_{4}^{2} \\
& +\left(\frac{R_{D 4}}{u}\right)^{3 / 2} u^{2} d \Omega_{4}^{2}
\end{aligned}
$$

where $u^{\prime}=d u / d x_{4}$. Just as for the low temperature phase, here too the CS term does not affect the solution of the equations of motion. Upon substituting the determinant of the induced metric and the dilaton, the DBI action is given by

$$
S_{D B I}=T_{8} \int d t d^{3} x d x_{4} d^{4} \Omega e^{-\phi} \sqrt{-\operatorname{det}(\hat{g})}=\frac{\hat{T}_{8}}{g_{s}} \int d x_{4} u^{4} \sqrt{f(u)} \sqrt{1+\left(\frac{R_{D 4}}{u}\right)^{3} \frac{u^{\prime 2}}{f(u)}},
$$

where $\hat{T}_{8}$ includes the outcome of the integration over all the coordinates apart from $d x_{4}$.

Again, conservation of the Hamiltonian of (24) implies that

$$
\frac{\sqrt{f(u)} u^{4}}{\sqrt{1+\left(\frac{R_{D 4}}{u}\right)^{3} \frac{u^{\prime 2}}{f(u)}}}=\text { constant }=u_{0}^{4} \sqrt{f\left(u_{0}\right)},
$$

where on the right-hand side of the equation we assumed that there is a point $u_{0}$ where the profile of the brane has a minimum, $u^{\prime}\left(u=u_{0}\right)=0$. Defining again $y \equiv u / u_{0}$, we 
(a)

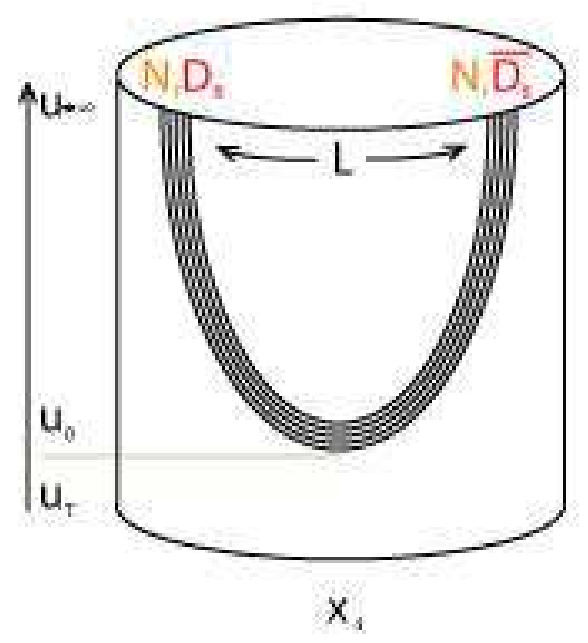

(b)

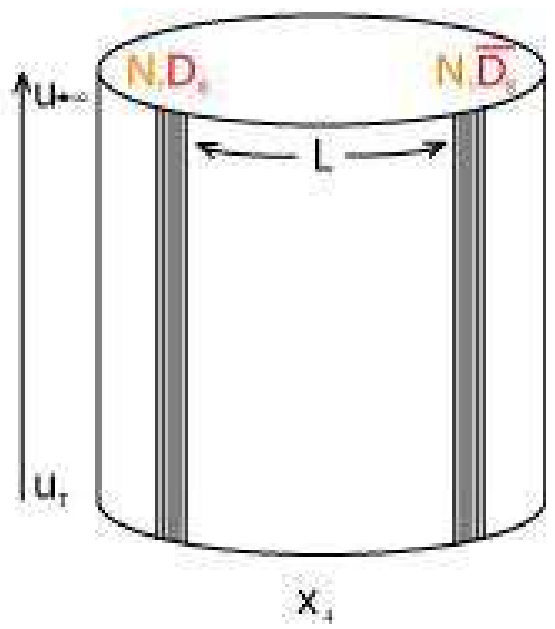

Figure 5: The possible configurations of the D8 and anti-D8 probe branes in the high temperature (deconfined) phase. A generic connected configuration with an asymptotic separation of $L$, that stretches down to a minimum at $u=u_{0}$, is drawn in (a). This corresponds to the chiral symmetry broken phase. Figure (b) depicts a chiral-invariant solution, with separate D8 and anti-D8-branes.

find that

$$
u^{\prime}=\left(\frac{u}{R_{D 4}}\right)^{3 / 2} \sqrt{f(y)} \sqrt{\frac{f(y)}{f(1)} y^{8}-1} .
$$

Substituting the outcome of the equation of motion, the action is now

$$
S_{D B I}=\frac{2 \hat{T}_{8} R_{D 4}^{3 / 2} u_{0}^{7 / 2}}{g_{s}} \int_{1}^{\infty} d y \frac{y^{5 / 2}}{\sqrt{1-\frac{f(1)}{f(y) y^{8}}}} .
$$

Since we assumed $u^{\prime}\left(u=u_{0}\right)=0$, this action corresponds to a configuration where the D8-branes merge smoothly into the anti-D8-branes, as in figure 5(a).

As discussed above, this configuration exhibits in the string theory dual the phenomenon of $\chi \mathrm{SB}$ in the gauge theory. However, there is yet another classical configuration that solves the equations of motion. This is a configuration of a constant $x_{4}$, $x_{4}(u)=0$ and $x_{4}(u)=L\left(\right.$ or $\left.u^{\prime} \rightarrow \infty\right)$, see figure 5(b). This configuration is possible in the high-temperature phase since in this phase the $t$ circle shrinks to zero at $u=u_{T}$, so the D8-brane can just smoothly end there (and wrap around the "cigar" in the $t-u$ 


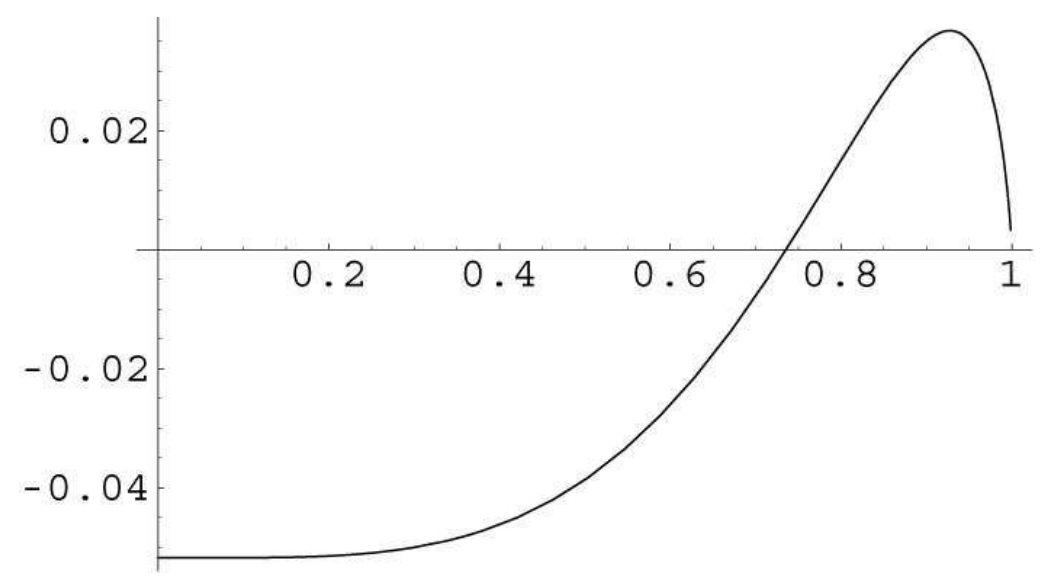

Figure 6: $\Delta S$ as a function of $y_{T}$, in units of $2 \hat{T}_{8} R_{D 4}^{3 / 2} u_{0}^{7 / 2} / g_{s}$.

plane); in the Minkowski space continuation of this solution, the D8-brane would go into the horizon of the black D4-branes. For such a configuration that naturally describes the situation of an unbroken chiral symmetry, the action reads (summing over the D8 and anti-D8 contributions)

$$
S_{D B I}^{0}=\frac{2 \hat{T}_{8}}{g_{s}} \int_{u_{T}}^{\infty} d u u^{4}\left(\frac{R_{D 4}}{u}\right)^{3 / 2}=\frac{2 \hat{T}_{8} R_{D 4}^{3 / 2} u_{0}^{7 / 2}}{g_{s}}\left[\int_{1}^{\infty} d y y^{5 / 2}+\int_{y_{T}}^{1} d y y^{5 / 2}\right],
$$

where $y_{T} \equiv u_{T} / u_{0}$.

We are now facing the question of which of the two possible configurations is preferred. This is determined by the free energy, or the action, of the configurations. The relevant quantity is the following difference between the two actions

$$
\begin{aligned}
\Delta S & \equiv \frac{g_{s}\left(S-S^{0}\right)}{2 \hat{T}_{8} R_{D 4}^{3 / 2} u_{0}^{7 / 2}}=\left\{\int_{1}^{\infty} d y y^{5 / 2}\left[\frac{1}{\sqrt{1-\frac{f(1)}{f(y)} y^{-8}}}-1\right]-\int_{y_{T}}^{1} d y y^{5 / 2}\right\} \\
& =\frac{1}{3}\left\{\int_{0}^{1} d z \frac{1}{z^{13 / 6}}\left[\frac{\sqrt{1-y_{T}^{3} z}}{\sqrt{1-y_{T}^{3} z-\left(1-y_{T}^{3}\right) z^{8 / 3}}}-1\right]\right\}-\frac{2}{7}\left(1-y_{T}^{7 / 2}\right) .
\end{aligned}
$$

We are unable to evaluate $\Delta S$ analytically so we do it numerically. The result is drawn in figure 6. which shows $\Delta S$ as a function of $y_{T}$. It is easy to see from this figure that for $y_{T}>y_{T}^{c} \sim 0.73572, \Delta S$ is positive and hence the preferred configuration is that of disconnected D8 and anti-D8-branes, whereas in the region where $y_{T}<y_{T}^{c} \sim 0.73572$ the system will be dominated by a smooth configuration that connects the D8 and anti D8 branes, namely the chiral symmetry broken phase. 
Next, we would like to express the critical point in terms of physical quantities. Recall that $y_{T} \equiv u_{T} / u_{0} . u_{T}$ is related to the temperature in the gauge theory by (22). $u_{0}$ is the minimal point of the connected probe brane configuration, which can be related to the asymptotic distance $L$ between the positions of the D8 and anti-D8-branes (see figure 5 ) in the following manner ${ }^{12}$

$$
\begin{aligned}
L & =\int d x_{4}=2 \int_{u_{0}}^{\infty} \frac{d u}{u^{\prime}}=2\left(\frac{R_{D 4}^{3}}{u_{0}}\right)^{1 / 2} \int_{1}^{\infty} d y \frac{y^{-3 / 2}}{\sqrt{f(y)} \sqrt{\frac{f(y)}{f(1)} y^{8}-1}} \\
& =\frac{2}{3}\left(\frac{R_{D 4}^{3}}{u_{0}}\right)^{1 / 2} \sqrt{1-y_{T}^{3}} \int_{0}^{1} d z \frac{z^{1 / 2}}{\sqrt{1-y_{T}^{3} z} \sqrt{1-y_{T}^{3} z-\left(1-y_{T}^{3}\right) z^{8 / 3}}} .
\end{aligned}
$$

Note that for small $L$, namely for $y_{T}<<1$, we find $u_{0} \propto R_{D 4}^{3} / L^{2}$ and again $S \propto 1 / L^{7}$. We would like to relate the asymptotic distance $L$ at the transition to the temperature of the transition. Evaluating the integral in (30) at the transition temperature $y_{T}^{c} \simeq 0.73572$ gives $L_{c} \simeq 0.751\left(R_{D 4}^{3} / u_{0}\right)^{1 / 2}$, and using equation (22) we find

$$
T_{\chi S B} \simeq 0.154 / L \text {. }
$$

Recall that the deconfinement phase transition is at $T_{d}=1 / 2 \pi R$, and that this analysis applies only in the deconfined phase. For $L>0.97 R$ the temperature in the deconfined phase is always higher than (31), so the deconfinement and chiral symmetry restoration transitions happen together. On the other hand, for $L<0.97 R$, the two phase transitions are separate, and there is a finite range between $T_{d}$ and $T_{\chi S B}$ for which the theory is deconfined but with a broken chiral symmetry. The full phase diagram of the theory (in the supergravity approximation) is drawn in figure 7 . Note that even though for the gravity approximation to be good everywhere in space we required $\lambda_{5} \gg R$, for gravity to be good near the 8-branes it is actually enough to require $\lambda_{5} \gg L$, and at high temperatures gravity gives a good approximation everywhere whenever $\lambda_{5} \gg 1 / T$. Thus, we can trust our analysis of the phase transition whenever $\lambda_{5} \gg L$. In particular, in the $R \rightarrow \infty$ limit discussed in [20], the theory is always deconfined, and passes through a chiral symmetry restoration transition at $T=T_{\chi S B}$ given by (31). ${ }^{13}$

\subsection{The spectrum in the various phases}

In this subsection we discuss the spectrum of quark configurations in the different phases described above; such a discussion is more appropriate in Minkowski space, in the

\footnotetext{
${ }^{12}$ In fact, as we discussed above, this integral could equal either $L$ or $2 \pi R-L$, but it is easy to see that the second option is always sub-dominant wherever it exists.

${ }^{13}$ Note added : in parallel with this paper, the paper 32] appeared which discusses the same system in the $R \rightarrow \infty$ limit, with identical results to ours.
} 


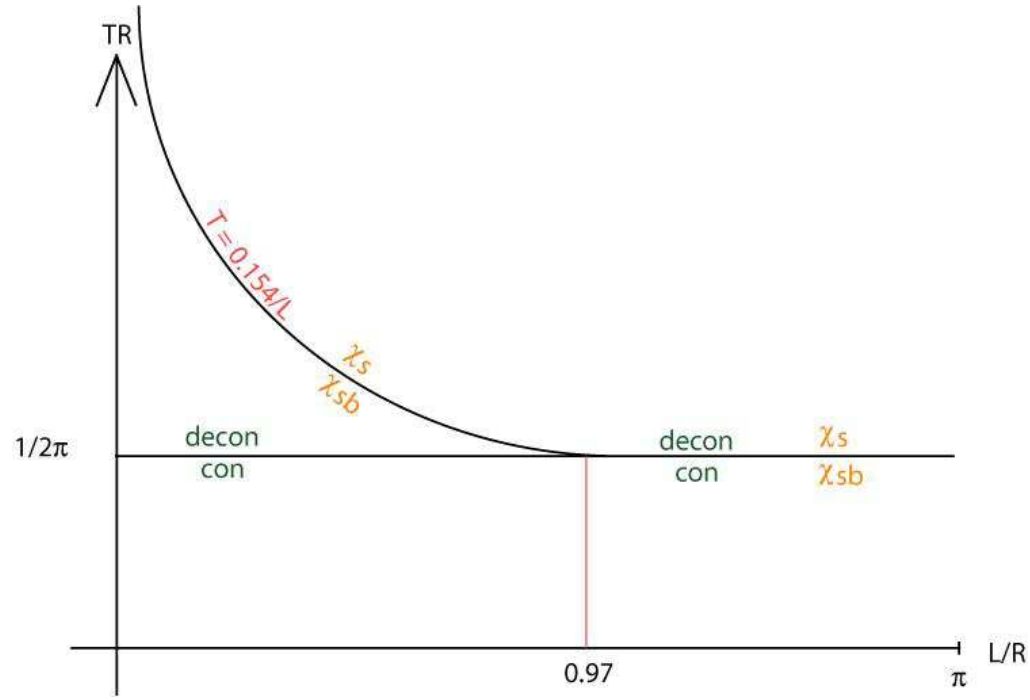

Figure 7: The phase diagram of the Sakai-Sugimoto model at finite temperature, in the gravity approximation $\lambda_{5} \gg R$. In this approximation the phase structure depends only on the two dimensionless parameters $T R$ and $L / R$. For small $L / R$ the deconfinement and chiral symmetry restoration transitions are separate, while for $L / R>0.97$ there is a single transition.

finite-energy-density configurations (in the microcanonical ensemble) obtained by Wickrotating the Euclidean configurations discussed above. In general there are two types of possible configurations. We can have strings with both ends on D8-branes, corresponding to mesons (for light strings these are described by the DBI action, and for large spin they can be described by semi-classical strings, see figure 2). And, if the background contains a horizon (as in the deconfined phase) we can have a string starting on an 8-brane and going into the horizon, corresponding to a deconfined quark (or anti-quark).

As discussed above, the behavior in the low-temperature phase is very similar to the behavior at zero temperature, as expected in a theory with a small number of weakly interacting massless particles (the "pions"). There is a discrete spectrum of mesons, and there are no free quark states. Computing the spectrum of mesons (including the pseudo vector and scalar mesons described in $\S 2.2$ ) and glueballs in this phase gives exactly the same results as for zero temperature. The high spin mesons are described as classical string configurations stretched from the D8 probe brane down to the "wall" at $u=u_{\Lambda}$ and back to the probe branes [15] (see figure 2), and again there is no difference between the zero temperature and low temperature results.

As we raise the temperature, at $T=1 / 2 \pi R$ we go over to the deconfined bulk 
phase; as we saw, depending on $L / R$, we can either get a phase with broken chiral symmetry or with unbroken chiral symmetry. In the case of small $L / R$ with broken chiral symmetry the D8-branes are still connected. In this phase the spectrum of bulk modes has no mass gap, due to the presence of the horizon in (21). On the other hand, a computation of the meson spectrum using the D8-brane action still gives a discrete spectrum as in [17]. However, unlike the low-temperature case, here the spectrum of quark-anti-quark configurations is continuous at high enough energies. This is because this spectrum includes strings starting on the D8-brane and going down to the horizon $u=u_{T}$ (in Minkowski space such configurations are just deconfined quarks, while in Euclidean space these strings must go back to the D8-brane, but they can stretch any distance in the three dimensional space before doing so with no cost in energy). These excitations give rise to a continuous spectrum of quark-anti-quark configurations, which starts above a mass gap which equals twice the mass of a string stretching from $u_{0}$ to $u_{T}$, $M_{\text {gap }}=2 \int_{u_{T}}^{u_{0}} \sqrt{1-\left(u_{T} / u\right)^{3}} d u / l_{s}^{2}$. This is obviously the analog of $2 m_{q}^{C}$ defined in (17), and this also scales as $\lambda_{5} / L^{2}$ for small $L / R$. Note that after we put in the D8-branes we can no longer use the spatial Wilson loop as a diagnostic for confinement, since this can be screened by the dynamical quarks, but the scaling of the free energy at large $N_{c}$ can still be used to distinguish the confining phase at $T<1 / 2 \pi R$ from the deconfined phase at $T>1 / 2 \pi R$.

Even when $L / R$ is small and such an intermediate phase exists, at high enough temperature we always end up in a deconfined phase with a restored chiral symmetry, in which the 8-branes end at the horizon $u=u_{T}$. In this phase there is no longer a mass gap for quark-anti-quark configurations, since strings stretched between the 8-branes and the horizon can be as light as we want, and the spectrum of quark-anti-quark configurations is continuous (just like the bulk spectrum).

\section{Summary}

In this paper we computed the phase structure of the Sakai-Sugimoto model, which is an example of a confining gauge theory with chiral symmetry breaking, related by a continuous change of parameters to large $N_{c}$ QCD. Our results are summarized in figure 7. We found that the topology of the solutions (in the supergravity approximation) implies that the confined phase must always break the chiral symmetry. On the other hand, in the deconfined phase a computation is required to see whether chiral symmetry is restored at the deconfinement temperature or above it, and we found that in some range of parameters the first possibility is realized, while in another range the second possibility occurs. When $L$ is much smaller than $R$ we found that the chiral symmetry restoration scale is much higher than the deconfinement scale, as expected because of 
the separation between the confinement and chiral symmetry breaking scales in this case (as emphasized in [20]). In this case the chiral symmetry restoration happens at a scale related to the mass scale of the mesons $1 / L$, while the deconfinement transition happens at a scale related to the glueball mass scale $1 / R$.

The fact that confinement implies chiral symmetry breaking is true also in a larger class of holographic duals of confining theories. When the confinement is realized geometrically (this is not always true [3]) there is a minimal value of the radial coordinate $u=u_{\Lambda}$, and some spatial cycle shrinks there. A chiral symmetry should be realized by having two sets of branes (each carrying one $U\left(N_{f}\right)$ factor), and chiral symmetry breaking should be realized by the branes becoming connected. If the branes can end without reconnecting, confinement does not imply $\chi \mathrm{SB}$; clearly this can only happen if the branes are wrapped on the cycle which vanishes at the minimal value $u=u_{\Lambda}$. Conversely, if the branes are not wrapped around this cycle (as is the case in the Sakai-Sugimoto model which we discussed here), they do not have anywhere to end, so the chiral symmetry must be broken in the confined phase. It would be interesting to relate this discussion to the gauge theory discussions of chiral symmetry breaking in large $N_{c}$ gauge theories [30, 31] which show that chiral symmetry must be broken in the confined phase in the large $N_{c}$ limit.

In this paper we discussed the phase structure of the theory only in the gravity regime $\lambda_{5} \gg L$. It was recently realized in [20] that there is another regime in which the theory is under control, which is the weak-coupling regime $\lambda_{5} \ll L$ with $R$ going to infinity. It would be interesting to study the phase structure also in this regime. There are also many other possible generalizations of the Sakai-Sugimoto model which can be analyzed by similar methods to the ones we used here, such as having several different stacks of D8-branes (and of anti-D8-branes) separated in the $x_{4}$ direction.

It is interesting to compare our results with the situation in large $N_{c} \mathrm{QCD}$, which is related to our theory by taking the limit of small $\lambda_{5} / R$. The technology to study large $N_{c}$ QCD with chiral fermions on the lattice has only recently been developed, and preliminary results presented in [33] suggest that in this theory chiral symmetry restoration occurs at the same temperature as the deconfinement transition, but that if one supercools the deconfined phase below the deconfinement temperature one encounters a critical temperature where the chiral symmetry is broken. This is similar to what we find in the case of $L / R>0.97$ (where we also find a transition in the supercooled deconfined phase at the temperature $T_{c}=0.154 / L$ ), so it is plausible that the phase diagram in this regime connects continuously to that of large $N_{c}$ QCD (up to the caveats discussed at the end of $\S 3.1$ ). 


\section{Acknowledgements}

We would like to thank S. Minwalla, H. Neuberger, B. Svetitsky, M. Van Raamsdonk and especially D. Kutasov for useful discussions. J.S. would like to thank O. Sonnenschein for helping with the drawings. This work was supported in part by a grant of DIP (H.52) and by the Albert Einstein Minerva Center for Theoretical Physics at the Weizmann Institute. The work of O.A. was supported in part by the Israel-U.S. Binational Science Foundation, by the Israel Science Foundation (grant number 1399/04), by the BraunRoger-Siegl foundation, by the European network HPRN-CT-2000-00122, by a grant from the G.I.F., the German-Israeli Foundation for Scientific Research and Development, and by Minerva. The work of J.S. and S.Y. was supported in part by the Israel Science Foundation (grant number 03200306).

\section{A Appendix: The bulk free energies of the low and high temperature phases}

In this appendix we present the calculation of the difference between the free energies of the two possible solutions for the finite temperature description of the bulk, described in section 3.1. A similar calculation in the context of the $A d S_{5} \times S^{5}$ model was done in [2]. Rather than computing the action directly, we translate our results into the notations of [34] and use the results for the action computed there. Thus, we parameterize the Euclidean metrics (10) and (21) that we are interested in as

$$
l_{s}^{-2} d s^{2}=d \tau^{2}+e^{2 \lambda(\tau)} d x_{\|}^{2}+e^{2 \tilde{\lambda}(\tau)} d x_{c}^{2}+e^{2 \nu(\tau)} d \Omega_{4}^{2},
$$

where $x_{c}$ is either $x_{4}$ or the Euclidean time (the one whose circle shrinks to zero size at the minimal value of $u$ ), $x_{\|}$are the other four coordinates of $R^{4,1}$ (one of which is also compactified), $\tau$ is the radial direction and $d \Omega_{4}^{2}$ is the metric on the transverse $S^{4}$. The background includes in addition the dilaton $\phi$ and a four-form RR field. For such backgrounds that depend only on a radial direction, the full type IIA supergravity action reduces to the following $0+1$-dimensional action [35] :

$$
S=V \int d \rho\left(-4\left(\lambda^{\prime}\right)^{2}-\left(\tilde{\lambda}^{\prime}\right)^{2}-4\left(\nu^{\prime}\right)^{2}+\left(\varphi^{\prime}\right)^{2}+12 e^{-2 \nu-2 \varphi}-Q_{c}^{2} e^{4 \lambda+\tilde{\lambda}-4 \nu-\varphi}\right),
$$

where the new radial coordinate $\rho$ is defined by $d \tau=-e^{-\varphi} d \rho$, primes denote derivatives with respect to $\rho$, and $\varphi \equiv 2 \phi-4 \lambda-\tilde{\lambda}-4 \nu . V$ is the volume of all other directions (except $\rho$ ) in string units; this includes the volume $V_{3}$ in the three spatial directions, the volume of the two circles which is $2 \pi R \beta / l_{s}^{2}$ and the volume of a unit $S^{4}$ which is 
$2 \pi^{2}$. The last term in the action corresponds to the contribution of the RR flux, with $Q_{c} \equiv 3 \pi N_{c} / \sqrt{2}$ (see, for instance, [19]) where $N_{c}$ is the quantized RR flux.

The equations of motion associated with the action (33) are

$$
\begin{aligned}
\lambda^{\prime \prime}-\frac{1}{2} Q_{c}^{2} e^{2(4 \lambda+\tilde{\lambda}-\phi)} & =0, \\
\tilde{\lambda}^{\prime \prime}-\frac{1}{2} Q_{c}^{2} e^{2(4 \lambda+\tilde{\lambda}-\phi)} & =0, \\
\phi^{\prime \prime}-\frac{1}{2} Q_{c}^{2} e^{2(4 \lambda+\tilde{\lambda}-\phi)} & =0, \\
\nu^{\prime \prime}-3 e^{2(4 \lambda+\tilde{\lambda}+3 \nu-2 \phi)}+\frac{1}{2} Q_{c}^{2} e^{2(4 \lambda+\tilde{\lambda}-\phi)} & =0 .
\end{aligned}
$$

It is straightforward to check that the following are the solutions of these equations [34, 36] corresponding to the background (11):

$$
\begin{array}{rlrl}
\lambda & =-\frac{1}{4} \ln (\sinh (-4 b \rho))+\frac{1}{4} \ln \left(\frac{u_{\Lambda}^{3}}{2 R_{D 4}^{3}}\right)-b \rho, & \tilde{\lambda} & =\lambda+4 b \rho, \\
\phi=\lambda+\ln \left(g_{s}\right), & \nu=\frac{\lambda}{3}+\ln \left(R_{D 4} / l_{s}\right),
\end{array}
$$

where $b \equiv-Q_{c} u_{\Lambda}^{3} / 4 \sqrt{2} R_{D 4}^{3} g_{s}$. Here we wrote the solution of the low temperature phase, for the high temperature phase we have to replace $u_{\Lambda}$ with $u_{T}$.

Since the backgrounds (11) and (21) are expressed in terms of the radial coordinate $u$, it is useful to rewrite the action (33) in terms of integrals over $u$, which run from $u_{\Lambda}$ to $\infty$ for the low temperature phase and from $u_{T}$ to $\infty$ for the high temperature phase. The action (33) then reads (with dots denoting derivatives with respect to $u$ )

$$
S=-V \int_{u_{\Lambda}}^{\infty} d u\left\{\left[-4 \dot{\lambda}^{2}-\dot{\tilde{\lambda}}^{2}-4 \dot{\nu}^{2}+\dot{\varphi}^{2}\right] \frac{d u}{d \rho}+\left[12 e^{8 \lambda+2 \tilde{\lambda}+6 \nu-4 \phi}-Q_{c}^{2} e^{8 \lambda+2 \tilde{\lambda}-2 \phi}\right] \frac{d \rho}{d u}\right\},
$$

where the minus sign arises because $d \rho / d u$ is negative. Substituting the solution (11), the action takes the form

$$
S=-V \int_{u_{\Lambda}}^{\infty} d u\left\{\left[\frac{15}{2 u^{2}}\left(1+\frac{1}{\left[\left(\frac{u}{u_{\Lambda}}\right)^{3}-1\right]}\right)\right] \frac{d u}{d \rho}+\left[\left(\frac{12}{g_{s}^{4} l_{s}^{6}}-\frac{Q_{c}^{2}}{g_{s}^{2} R_{D 4}^{6}}\right) u^{6} f(u)\right] \frac{d \rho}{d u}\right\} .
$$

At this point we need to compute the derivative $\frac{d \rho}{d u}$ and its inverse. Using the relation $e^{2 \tilde{\lambda}} / e^{2 \lambda}=\left[1-\left(\frac{u_{\Lambda}}{u}\right)^{3}\right]$ and (35) we find that $\frac{d \rho}{d u}$ is given by

$$
\frac{d \rho}{d u}=\frac{3}{8 b u} \frac{1}{\left[\left(\frac{u}{u_{\Lambda}}\right)^{3}-1\right]} .
$$


Substituting this expression into the action we find that the divergence at large $u$ is independent of $u_{\Lambda}$, so it makes sense to subtract the expressions with $u_{\Lambda}$ and with $u_{T}$ and to obtain a finite answer. The result for the difference between the action densities in the low temperature phase and in the high temperature phase is given by (defining $\hat{b}=b l_{s}^{3} / u_{\Lambda}^{3}$ which is a constant independent of $u_{\Lambda}$ )

$$
\begin{aligned}
\frac{\Delta S}{V_{3}} \equiv \frac{S_{\text {low }}-S_{\text {high }}}{V_{3}} & =\frac{4 \pi^{3} R \beta}{l_{s}^{8}}\left[\frac{20 \hat{b}}{3}+\frac{1}{4 \hat{b}}\left(\frac{6}{g_{s}^{4}}-\frac{Q_{c}^{2}}{2 g_{s}^{2} R_{D 4}^{6}}\right)\right]\left(u_{\Lambda}^{3}-u_{T}^{3}\right) \\
& =\frac{4 \pi^{3} R \beta}{l_{s}^{8}} \frac{5}{g_{s}^{2}}\left(u_{T}^{3}-u_{\Lambda}^{3}\right) \\
& =20 \beta R l_{s}\left(\frac{4}{9}\right)^{3} \pi^{6}\left(g_{s} N_{c}\right) N_{c}^{2}\left[-\frac{1}{R^{6}}+\frac{1}{\left(\frac{\beta}{2 \pi}\right)^{6}}\right] \\
& =20 \beta R l_{s}\left(\frac{4}{9}\right)^{3} \pi^{6}\left(g_{s} N_{c}\right) N_{c}^{2}\left[(2 \pi T)^{6}-M_{g b}^{6}\right] .
\end{aligned}
$$

In the third and fourth lines the difference of the actions was expressed in terms of the radii of the circles and in terms of the glueball mass scale and the temperature, respectively. The difference of actions scales as $N_{c}^{2}$ in the 't Hooft large $N_{c}$ limit, as expected.

The difference (39) between the action densities is the same, up to a factor of $\beta$, as the difference between the free energy densities. It is now easy to see that for low temperatures, $2 \pi R<\beta$, the background (11) has a lower free energy so it dominates, while the opposite is the case for high temperatures, as we claimed in section 3.1.

\section{References}

[1] J. M. Maldacena, The large $N$ limit of superconformal field theories and supergravity, Adv. Theor. Math. Phys. 2, 231 (1998) [Int. J. Theor. Phys. 38, 1113 (1999)] arXiv:hep-th/9711200.

[2] E. Witten, Anti-de Sitter space, thermal phase transition, and confinement in gauge theories, Adv. Theor. Math. Phys. 2, 505 (1998); hep-th/9803131.

[3] J. Polchinski and M. J. Strassler, The string dual of a confining four-dimensional gauge theory, arXiv:hep-th/0003136.

[4] I. R. Klebanov and M. J. Strassler, Supergravity and a confining gauge theory: Duality cascades and chiSB-resolution of naked singularities, JHEP 0008, 052 (2000); hep-th/0007191. 
[5] J. M. Maldacena and C. Nunez, Towards the large $N$ limit of pure $N=1$ super Yang Mills, Phys. Rev. Lett. 86, 588 (2001); hep-th/0008001.

[6] A. Karch and A. Katz, Adding flavor to AdS/CFT, JHEP 0206, 043 (2002); hep-th/0205236.

[7] O. Aharony, A. Fayyazuddin and J. M. Maldacena, The large $N$ limit of $N$ = 2,1 field theories from three-branes in F-theory, JHEP 9807, 013 (1998) arXiv:hep-th/9806159.

[8] T. Sakai and J. Sonnenschein, Probing flavored mesons of confining gauge theories by supergravity, JHEP 0309, 047 (2003); hep-th/0305049.

[9] J. Babington, J. Erdmenger, N. J. Evans, Z. Guralnik and I. Kirsch, Chiral symmetry breaking and pions in non-supersymmetric gauge / gravity duals, Phys. Rev. D 69, 066007 (2004); hep-th/0306018.

[10] M. Kruczenski, D. Mateos, R. C. Myers and D. J. Winters, Towards a holographic dual of large-N(c) QCD, JHEP 0405, 041 (2004); hep-th/0311270.

[11] S. A. Cherkis and A. Hashimoto, Supergravity solution of intersecting branes and AdS/CFT with flavor, JHEP 0211 (2002) 036 arXiv:hep-th/0210105|; A. Karch, E. Katz and N. Weiner, Hadron masses and screening from AdS Wilson loops, Phys. Rev. Lett. 90 (2003) 091601 arXiv:hep-th/0211107. M. Kruczenski, D. Mateos, R. C. Myers and D. J. Winters, Meson spectroscopy in AdS/CFT with flavour, JHEP 0307, 049 (2003) hep-th/0304032; H. Nastase, On Dp-Dp+4 systems, QCD dual and phenomenology, hep-th/0305069; X. J. Wang and S. Hu, Intersecting branes and adding flavors to the Maldacena-Nunez background, JHEP 0309 (2003) 017; hep-th/0307218; P. Ouyang, Holomorphic D\%-branes and flavored $N=1$ gauge theories, Nucl. Phys. B 699 (2004) 207 arXiv:hep-th/0311084; C. Nunez, A. Paredes and A. V. Ramallo, Flavoring the gravity dual of $N=1$ Yang-Mills with probes, JHEP 0312, 024 (2003); hep-th/0311201; S. Hong, S. Yoon and M. J. Strassler, Quarkonium from the fifth dimension, JHEP 0404 (2004) 046 arXiv:hep-th/0312071; N. J. Evans and J. P. Shock, Chiral dynamics from AdS space, Phys. Rev. D 70 (2004) 046002 arXiv:hep-th/0403279; J. L. F. Barbon, C. Hoyos, D. Mateos and R. C. Myers, The holographic life of the eta', JHEP 0410 (2004) 029 arXiv:hep-th/0404260; M. Bando, T. Kugo, A. Sugamoto and S. Terunuma, Pentaquark baryons in string theory, Prog. Theor. Phys. 112 (2004) 325 arXiv:hep-ph/0405259; B. A. Burrington, J. T. Liu, L. A. Pando Zayas and D. Vaman, Holographic duals of flavored $N=1$ super Yang-Mills: Beyond the probe approximation, JHEP 0502, 022 (2005) arXiv:hep-th/0406207; 
K. Ghoroku and M. Yahiro, Chiral symmetry breaking driven by dilaton, Phys. Lett. B 604 (2004) 235 arXiv:hep-th/0408040; J. Erdmenger and I. Kirsch, Mesons in gauge / gravity dual with large number of fundamental fields, JHEP 0412 (2004) 025 arXiv:hep-th/0408113; D. Arean, D. Crooks and A. V. Ramallo, Supersymmetric probes on the conifold, JHEP 0411 (2004) 035 arXiv:hep-th/0408210; S. Hong, S. Yoon and M. J. Strassler, On the couplings of vector mesons in AdS/QCD, arXiv:hep-th/0409118; S. Hong, S. Yoon and M. J. Strassler, Adjoint trapping: A new phenomenon at strong 't Hooft coupling, JHEP 0603 (2006) 012 arXiv:hep-th/0410080; S. Kuperstein, Meson spectroscopy from holomorphic probes on the warped deformed conifold, JHEP 0503 (2005) 014 arXiv:hep-th/0411097; A. Paredes and P. Talavera, Multiflavour excited mesons from the fifth dimension, Nucl. Phys. B 713, 438 (2005) arXiv:hep-th/0412260; G. F. de Teramond and S. J. Brodsky, The hadronic spectrum of a holographic dual of $Q C D$, Phys. Rev. Lett. 94, 201601 (2005), hep-th/0501022; S. Hong, S. Yoon and M. J. Strassler, On the couplings of the rho meson in $A d S / Q C D$, arXiv:hep-ph/0501197; L. Da Rold and A. Pomarol, Chiral symmetry breaking from five dimensional spaces, Nucl. Phys. B 721, 79 (2005), hep-ph/0501218; K. Ghoroku, T. Sakaguchi, N. Uekusa and M. Yahiro, Flavor quark at high temperature from a holographic model, Phys. Rev. D 71 (2005) 106002 arXiv:hep-th/0502088; N. Evans, J. Shock and T. Waterson, D'7 brane embeddings and chiral symmetry breaking, JHEP 0503 (2005) 005 arXiv:hep-th/0502091; I. Brevik, K. Ghoroku and A. Nakamura, Meson mass and confinement force driven by dilaton, Int. J. Mod. Phys. D 15 (2006) 57 arXiv:hep-th/0505057; I. Kirsch and D. Vaman, The D3/Dr background and flavor dependence of Regge trajectories, Phys. Rev. D 72, 026007 (2005) arXiv:hep-th/0505164; T. S. Levi and P. Ouyang, Mesons and flavor on the conifold, arXiv:hep-th/0506021; N. Mahajan, Revisiting 5D chiral symmetry breaking and holographic QCD models, Phys. Lett. B 623, 119 (2005), hep-ph/0506098; K. Ghoroku, Flavor meson localization in 5d braneworld, Phys. Lett. B 632 (2006) 405 arXiv:hep-th/0506168; R. Apreda, J. Erdmenger and N. Evans, Scalar effective potential for D'7 brane probes which break chiral symmetry, hep-th/0509219; F. Canoura, J. D. Edelstein, L. A. P. Zayas, A. V. Ramallo and D. Vaman, Supersymmetric branes on $A d S_{5} \times Y^{p, q}$ and their field theory duals, JHEP 0603 (2006) 101 arXiv:hep-th/0512087; J. P. Shock, Canonical coordinates and meson spectra for scalar deformed $N=4 S Y M$ from the AdS/CFT correspondence, arXiv:hep-th/0601025 R. Casero, C. Nunez and A. Paredes, Towards the string dual of $N=1$ SQCD-like theories, arXiv:hep-th/0602027; T. Hirayama, A holographic dual of CFT with flavor on de Sitter space, arXiv:hep-th/0602258.

[12] M. Kruczenski, L. A. Pando Zayas, J. Sonnenschein and D. Vaman, Regge trajectories 
for mesons in the holographic dual of large-N(c) QCD, JHEP 0506, 046 (2005); hep-th/0410035.

[13] D. Bak and H. U. Yee, Separation of spontaneous chiral symmetry breaking and confinement via AdS/CFT correspondence, Phys. Rev. D 71 (2005) 046003 arXiv:hep-th/0412170.

[14] R. Casero, A. Paredes and J. Sonnenschein, Fundamental matter, meson spectroscopy and non-critical string / gauge duality, JHEP 0601, 127 (2006) arXiv:hep-th/0510110.

[15] K. Peeters, J. Sonnenschein and M. Zamaklar, Holographic decays of largespin mesons, JHEP 0602, 009 (2006) arXiv:hep-th/0511044; A. L. Cotrone, L. Martucci and W. Troost, String splitting and strong coupling meson decay, arXiv:hep-th/0511045.

[16] J. H. Brodie and A. Hanany, Type IIA superstrings, chiral symmetry, and $N=14 D$ gauge theory dualities, Nucl. Phys. B 506, 157 (1997) arXiv:hep-th/9704043.

[17] T. Sakai and S. Sugimoto, Low energy hadron physics in holographic QCD, Prog. Theor. Phys. 113, 843 (2005); hep-th/0412141.

[18] T. Sakai and S. Sugimoto, More on a holographic dual of QCD; hep-th/0507073.

[19] N. Itzhaki, J. M. Maldacena, J. Sonnenschein and S. Yankielowicz, Supergravity and the large $N$ limit of theories with sixteen supercharges, Phys. Rev. D 58, 046004 (1998) arXiv:hep-th/9802042.

[20] E. Antonyan, J. A. Harvey, S. Jensen and D. Kutasov, NJL and QCD from string theory, arXiv:hep-th/0604017.

[21] A. Brandhuber, N. Itzhaki, J. Sonnenschein and S. Yankielowicz, Wilson loops, confinement, and phase transitions in large $N$ gauge theories from supergravity, JHEP 9806, 001 (1998); hep-th/9803263

[22] Y. Kinar, E. Schreiber and J. Sonnenschein, $Q$ anti- $Q$ potential from strings in curved spacetime: Classical results, Nucl. Phys. B 566, 103 (2000); hep-th/9811192.

[23] S. R. Coleman and E. Witten, Chiral symmetry breakdown in large $N$ chromodynamics, Phys. Rev. Lett. 45, 100 (1980).

[24] F. Wilczek, Diquarks as inspiration and as objects, arXiv:hep-ph/0409168. 
[25] J. M. Maldacena and A. Strominger, AdS(3) black holes and a stringy exclusion principle, JHEP 9812 (1998) 005 arXiv:hep-th/9804085.

[26] J. M. Maldacena, Wilson loops in large $N$ field theories, Phys. Rev. Lett. 80, 4859 (1998) arXiv:hep-th/9803002.

[27] S. J. Rey and J. T. Yee, Macroscopic strings as heavy quarks in large $N$ gauge theory and anti-de Sitter supergravity, Eur. Phys. J. C 22, 379 (2001) arXiv:hep-th/9803001.

[28] O. Aharony, S. Minwalla and T. Wiseman, unpublished.

[29] O. Aharony, J. Marsano, S. Minwalla, K. Papadodimas, M. Van Raamsdonk and T. Wiseman, The phase structure of low dimensional large $N$ gauge theories on tori, JHEP 0601, 140 (2006) arXiv:hep-th/0508077.

[30] F. Neri and A. Gocksch, Chiral symmetry restoration in large $N Q C D$ at finite temperature, Phys. Rev. D 28, 3147 (1983).

[31] R. D. Pisarski, Finite temperature QCD at large N, Phys. Rev. D 29, 1222 (1984).

[32] A. Parnachev and D. A. Sahakyan, Chiral phase transition from string theory, arXiv:hep-th/0604173.

[33] R. Narayanan and H. Neuberger, Phases of planar QCD on the torus, PoS LAT2005 (2005) 005 arXiv:hep-lat/0509014.

[34] S. Kuperstein and J. Sonnenschein, Non-critical supergravity (d >1) and holography, JHEP 0407, 049 (2004) arXiv:hep-th/0403254.

[35] I. R. Klebanov and A. A. Tseytlin, D-branes and dual gauge theories in type 0 strings, Nucl. Phys. B 546 (1999) 155 arXiv:hep-th/9811035.

[36] S. Kuperstein and J. Sonnenschein, Non-critical, near extremal AdS $S_{6}$ background as a holographic laboratory of four dimensional YM theory, JHEP 0411, 026 (2004); hep-th/0411009. 